\title{
Efeito do fogo no banco de sementes do solo de sistemas agroflorestais, Pará, Brasil
}

\author{
Effect of fire in seed banks of agroforestry systems, Pará, Brazil \\ Fabrízia de Oliveira Alvino-Rayol ${ }^{1}$ e Breno Pinto Rayol ${ }^{2 *}$ \\ ${ }^{1}$ Instituto Federal de Educação, Ciência e Tecnologia do Pará, Belém, PA, Brasil. \\ ${ }^{2}$ Universidade Federal Rural da Amazônia, Belém, PA, Brasil. Autor para correspondência: bprayol@yahoo.com.br.
}

Submissão:17/07/2018 / Aceite: 01/07/2019

\begin{abstract}
RESUMO
O objetivo deste estudo foi avaliar o efeito do fogo na estrutura, composição florística e diversidade do banco de sementes do solo em diferentes sistemas agroflorestais (SAF). Foram avaliados quatro sistemas: a) SAF 1 - paricá (Schizolobium amazonicum) e caupi (Vigna unguiculata) com uso do fogo para o preparo da área; b) SAF 2 - paricá e caupi, feijão de porco (Canavalia ensiformes) sem uso do fogo; c) SAF 3 - paricá, açaí (Euterpe oleracea) e cupuaçu (Theobroma grandiflorum) com o uso do fogo; d) SAF 4 - paricá, açaí e cupuaçu, com preparo da área sem queima. O banco de sementes dos sistemas estudados foi avaliado através da coleta de 20 amostras de $50 \times 50 \mathrm{~cm}$ na camada de $0-5 \mathrm{~cm}$, totalizando 80 amostras. Para a contagem e identificação das espécies do banco de sementes, utilizouse a técnica de emergência de plântulas em casa de vegetação. A maior riqueza e diversidade foram encontradas no SAF 2. As espécies mais importantes foram Cyperus laxus, Olyra glaberrima e Myrciaria tenella. Os bancos de sementes das áreas que foram manejadas com fogo foram constituídos principalmente por espécies herbáceas. O fogo influenciou na florística, estrutura, diversidade do banco de sementes nos sistemas agroflorestais avaliados.
\end{abstract}

PALAVRAS-CHAVE: queima, solo, sementes.

\begin{abstract}
The objective of this study was to evaluate the effect of fire on floristic composition, structure, and diversity of seed banks in different agroforestry systems (SAF). We evaluated four systems: SAF 1 - Schizolobium amazonicum and Vigna unguiculata using fire to prepare the plot; b) SAF 2 - Schizolobium amazonicum, Vigna unguiculata and Canavalia ensiformes without the use of fire; c) SAF 3 - Schizolobium amazonicum, Euterpe oleracea and Theobroma grandiflorum with the use of fire; com; d) SAF 4 - Schizolobium amazonicum, Euterpe oleraceae and Theobroma grandiflorum without the use of fire. The seed bank was determined from 20 soil samples from $50 \times 50 \mathrm{~cm}$ at $0-5 \mathrm{~cm}$ depth level, totalizing 80 samples. For seed counting and identification, seedling emergence technique under greenhouse conditions was used. The most important species were Cyperus Laxus, Olyra glaberrima, and Myrciaria tenella. The seed banks of the agroforestry systems that were managed with fire were basically herbaceous species typical of agricultural lands and disturbed environments. Fire influenced the floristic, structure, diversity, and infestation of seed banks in agroforestry systems evaluated.
\end{abstract}

KEYWORDS: burn, soil, seeds.

\section{INTRODUÇÃO}

O armazenamento de sementes no solo é uma estratégia reprodutiva importante para as plantas, pois permite a colonização de habitats que estão sujeitos a distúrbios temporários ou periódicos (AULD \& DENHAM 2006). De acordo com UHL et al. (1981), as plântulas emergentes do banco de sementes reduzem a erosão e a perda de nutrientes após distúrbios, colaborando para a estabilização de áreas perturbadas. Assim, o banco de sementes pode ser uma importante fonte de reestabelecimento da vegetação após algum distúrbio (PARKER et al. 1989), desempenhando um papel fundamental no equilíbrio dinâmico da área (SCHIMTZ 1992).

Informações sobre as características dos bancos de sementes, como número de espécies, 
quantidade de sementes e distribuição destas no perfil do solo, permitem projeções sobre a composição florística dos estágios iniciais da regeneração e pode fornecer uma estimativa do potencial da regeneração após distúrbios (THOMPSON \& GRIME 1979, GARWOOD 1989). Portanto, o conhecimento a cerca da composição e densidade do banco de sementes do solo fornece indícios sobre a dinâmica, regeneração e restauração de ecossistemas degradados após distúrbios (GERHARDT \& HYTTEBORN 1992).

O impacto do fogo sobre a floresta faz retornar a vegetação aos estágios sucessionais iniciais, simplificando a composição de espécies e sua estrutura (LOUZADA et al. 2003). O regime do fogo afeta também processos demográficos como mortalidade, reprodução, germinação e sobrevivência de populações de plantas (WHELAN et al. 2002). Todos esses processos podem ser influenciados pelo fogo que pode aumentar ou restringir a produção de sementes, afetar os agentes dispersores, causar mortalidade e quebrar a dormência das sementes (KEITH 1996, WHELAN et al. 2002).

Compreende-se, então, a relevância deste estudo, quando se considera a importância ecológica do banco de sementes na regeneração e manutenção da vegetação espontânea e a sua vulnerabilidade perante o distúrbio de um fogo. O objetivo deste estudo foi avaliar o efeito do fogo no banco de sementes de sistemas agroflorestais no nordeste paraense.

\section{MATERIAL E MÉTODOS}

O presente estudo foi desenvolvido no município de Bragança, nordeste do estado do Pará. A região é caracterizada por extensas áreas de vegetação secundária, conhecidas localmente como capoeiras, em vários estágios de desenvolvimento, inclusive com muitas áreas já degradadas. O clima da região apresenta temperatura média anual de 25 a $26{ }^{\circ} \mathrm{C}$, pluviosidade de 2.200 a $3.000 \mathrm{~mm}$ por ano e umidade relativa média de $85 \%$, com solos ácidos de boa drenagem (IBGE 1983).

Os ensaios com sistemas agroflorestais foram conduzidos na Comunidade Benjamim Constant, a $28 \mathrm{~km}$ a sudeste da sede do município de Bragança, em propriedades agrícolas de produtores familiares, com predominância de vegetação secundária, anteriormente utilizadas com agricultura de subsistência no sistema de corte-queima.

Foi utilizada amostragem inteiramente casualizada, sendo a área experimental constituída de $19.764 \mathrm{~m}^{2}$, dividida em quatro subáreas: a) SAF $1\left(6.480 \mathrm{~m}^{2}\right)$ : paricá (Schizolobium amazonicum Huber) e caupi (Vigna unguiculata L.) com o uso do fogo para limpeza e preparo da área; b) SAF $2\left(6.480 \mathrm{~m}^{2}\right)$ : paricá, caupi e feijão de porco (Canavalia ensiformes) sem uso do fogo; c) SAF 3 (3.402 m²): paricá, açaí (Euterpe oleraceae Mart) e cupuaçu (Theobroma grandiflorum Schum), com o uso do fogo; d) SAF 4 (3.402 $\mathrm{m}^{2}$ ): paricá, açaí e cupuaçu, manejo sem queima. Os sistemas agroflorestais foram separados entre si por uma faixa de vegetação de capoeira de $10 \mathrm{~m}$ de largura.

Foram coletadas em cada área 20 amostras de solo, com auxílio de um gabarito de madeira medindo $0,5 \mathrm{~m} \times 0,5 \mathrm{~m}$, na profundidade de $0-5 \mathrm{~cm}$ sobre as quais foi adicionada a serapilheira correspondente, totalizando $5 \mathrm{~m}^{2}$ amostrados em cada área. Utilizou-se o sistema aleatório, com amostras distribuídas por toda a área. O material coletado foi acondicionado em saco plástico e transportado para uma casa de vegetação do Museu Paraense Emílio Goeldi, localizado na cidade de Belém. Em 80 bandejas plásticas $(45 \times 30 \times 7 \mathrm{~cm})$, preenchidas com vermiculita foram colocadas as amostras do solo, uma em cada bandeja, sendo irrigadas diariamente. Para verificar a ocorrência de contaminação foram mantidas na casa de vegetação oito bandejas, correspondentes a $10 \%$ das amostras, somente com vermiculita. Assim como realizado por NÓBREGA et al. (2009), duas vezes no decorrer do experimento, as bandejas foram mudadas de lugar para que as variações nas condições ambientais não influenciassem a germinação. A contagem e identificação das espécies foram realizadas em intervalos de 30 a 40 dias, durante 10 meses, conforme a velocidade de germinação das sementes. Após três meses o solo foi revolvido para que novos fluxos de emergência pudessem ocorrer.

As plântulas foram identificadas e comparadas às exsicatas do herbário de Museu Paraense Emílio Goeldi. A nomenclatura dos nomes científicos foi conferida utilizando as bases de dados do Missouri Botanical Garden (http://mobot.bobot.org/W3T/Search/vas.html). As amostras de material botânico não identificado receberam códigos de morfotipo (material não identificado taxonomicamente) e foram consideradas como espécies diferentes entre si, mesmo quando pertencentes ao mesmo gênero.

Os valores estimados de densidade de sementes germinadas e riqueza de espécies foram comparados entre os diferentes SAF's por meio de análise de variância, após serem submetidos à transformação por log $(n+1)$. O total de sementes foi obtido pela soma de todas as plântulas emergidas, em cada amostra, durante esse período.

A riqueza, definida como número de espécies presentes em uma determinada área, foi estimada por 
meio da contagem das espécies do banco de sementes que ocorreram em cada SAF. A composição florística dos bancos de sementes foi avaliada através da riqueza (S) e índice de diversidade de ShannonWiener (H') (MAGURRAN 1988). As espécies foram avaliadas através dos parâmetros de densidade absoluta e relativa, frequência absoluta e relativa e índice de importância modificado (IM) para cada espécie correspondente a soma da densidade relativa e frequência relativa (MUELLER-DOMBOIS \& ELLENBERG 1974).

As espécies identificadas foram classificadas quanto à forma de vida (hábito), nas seguintes categorias: árvore, arbusto, ervas ou gramíneas e lianas conforme adotado por outros autores (UHL \& BUSCHBACHER 1985, LORENZI 2006).

\section{RESULTADOS}

O sistema agroflorestal SAF 3 onde foi utilizado fogo no preparo da área obteve os menores valores de densidade (88 sementes $\left./ \mathrm{m}^{2}\right)$, riqueza $(25)$ e diversidade $(1,26)$ quando comparados com os demais sistemas avaliados. O SAF 2 (sem queima) apresentou maior riqueza de espécies (45), ao passo que os sistemas que sofreram queima apresentaram perda no número de espécies quando comparados às áreas não atingidas pela queima (Tabela 1). Quando comparados, a perda de espécies no SAF 3 foi cerca de $22 \%$ em relação ao SAF 4 (sem queima).

Tabela 1. Densidade (DA), riqueza (S), Índice de diversidade de Shannon-Wiener ( $\left.H^{\prime}\right)$ do banco de sementes do solo de sistemas agroflorestais, Pará, Brasil. (SAF 1 - paricá e caupi com uso do fogo para o preparo da área; SAF 2 - paricá, caupi e feijão de porco sem uso do fogo; SAF 3 - paricá, açaí e cupuaçu com o uso do fogo; SAF 4 - paricá, açaí e cupuaçu, sem uso do fogo).

Table 1. Density (DA), richness (S), Index Shannon-Wiener $\left(H^{\prime}\right)$ of seed bank of soil agroforestry systems, Pará, Brazil. (SAF 1 - Schizolobium amazonicum and Vigna unguiculata using fire to prepare the plot; SAF 2 - S. amazonicum, V.unguiculata and Canavalia ensiformes without the use of fire; SAF 3 - S.amazonicum, Euterpe oleracea and Theobroma grandiflorum with the use fire; com; SAF 4S. amazonicum, E. oleraceae and T. grandiflorum without the use of fire).

\begin{tabular}{cccc}
\hline Sistemas & DA $\left(\right.$ sementes $/ \mathrm{m}^{2)}$ & $\mathrm{S}$ & $\mathrm{H}^{\prime}$ \\
\hline SAF 1 & $104 \mathrm{a}$ & 32 & $1,3198 \mathrm{~b}$ \\
SAF 2 & $105 \mathrm{a}$ & 45 & $1,4743 \mathrm{a}$ \\
SAF 3 & $88 \mathrm{c}$ & 25 & $1,2598 \mathrm{c}$ \\
SAF 4 & $100 \mathrm{~b}$ & 32 & $1,3734 \mathrm{~b}$ \\
\hline
\end{tabular}

Nos bancos de sementes das áreas que foram preparadas com fogo, SAF1 e SAF3, as formas de vida predominantes foram as ervas e lianas (SAF $1-50 \%$, SAF $3-55 \%$ ). Enquanto que nas áreas dos sistemas agroflorestais que não sofreram a ação do fogo durante 0 preparo do solo, verificou-se 0 predomínio das espécies arbóreas e arbustivas (SAF 2 - 60\%; SAF 4 - 65\%) (Figura 1).

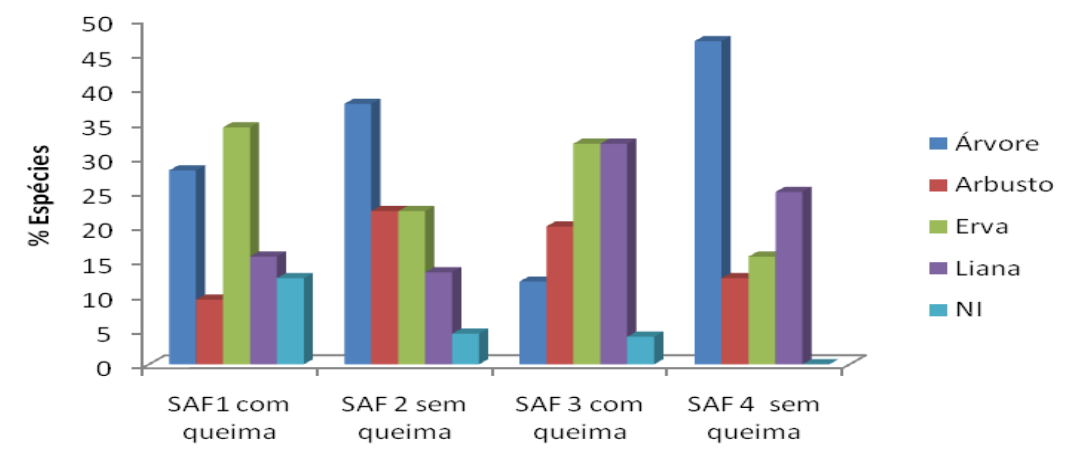

Figura 1. Percentual de espécies por forma de vida, germinadas no banco de sementes do solo de sistemas agroflorestais, Pará, Brasil. (SAF 1 - paricá e caupi com uso do fogo para o preparo da área; SAF 2 - paricá, caupi e feijão de porco sem uso do fogo; SAF 3 - paricá, açaí e cupuaçu com o uso do fogo; SAF 4 - paricá, açaí e cupuaçu, sem uso do fogo; $\mathrm{NI}$ - Não identificada).

Figure 1. Percentage of species by life form, germinated in the seed bank of soil of agroforestry systems, Pará, Brazil. (SAF 1 - Schizolobium amazonicum and Vigna unguiculata using fire to prepare the plot; SAF 2 - S. amazonicum, V.unguiculata and Canavalia ensiformes without the use of fire; SAF 3 - S.amazonicum, Euterpe oleracea and Theobroma grandiflorum with the use fire; com; SAF 4 S. amazonicum, E. oleraceae and T. grandiflorum without the use of fire; NI - Not identified). 
A florística do banco de sementes nos quatro SAF's estudados apresentou 30 famílias, 77 espécies e seis plântulas não identificadas que foram classificadas como morfotipo. No SAF1 emergiram 32 espécies, distribuídas em 20 famílias do banco de sementes das amostras coletadas; no SAF2 45 espécies distribuídas em 24 famílias; no SAF3 25 espécies distribuídas em 19 famílias e no SAF4 32 com 17 famílias. As famílias com maior número de espécies foram: Fabaceae com nove espécies; Cyperaceae e Dilleniaceae, cada uma com seis espécies; Euphorbiaceae e Melastomataceae, cinco espécies cada; Myrtaceae, Poaceae e Rubiaceae com quatro espécies cada; Malvaceae com três espécies e as demais com duas ou uma espécie cada.

As espécies mais importantes no banco de sementes do SAF1 foram: Cyperus laxus, Olyra glaberrima, Rhynchospora rubber e Doliocarpus brevipedicelatta (Tabela 2). Em termos de densidade, Cyperus laxus lidera com 15,11\%, seguida de Olyra glaberrima (12,05\%). A queima condicionou um comportamento diferenciado da estrutura do banco de sementes. Observa-se no grupo das ciperáceas altos valores relativos de densidade e freqüência.

Tabela 2. Percentual dos parâmetros fitossociológicos das espécies do banco de sementes do solo nos SAF 1 (paricá e caupi com uso do fogo para o preparo da área), Pará, Brasil. ( $D R=$ Densidade Relativa, FR = Frequência Relativa, IM = Índice de Valor de Importância Modificado).

Table 2. Percentage of phytosociological parameters of species seed bank of soil in the agroforestry system 1 (Schizolobium amazonicum and Vigna unguiculata using fire to prepare the plot), Pará, Brazil. ( $D R=$ Relative Density, $F R=$ Relative Frequency, $I M=$ Importance Value Index Modified).

\begin{tabular}{|c|c|c|c|}
\hline Espécie & DR & FR & IM \\
\hline Cyperus laxus Lam. & 15,11 & 9,09 & 24,20 \\
\hline Olyra glaberrima Raddi & 12,05 & 7,14 & 19,19 \\
\hline Rhynchospora sp. & 8,03 & 7,14 & 15,17 \\
\hline Doliocarpus brevipedicellatus Garcke & 7,65 & 5,84 & 13,49 \\
\hline Lecythis lurida (Miers) S.A. Mori & 4,21 & 6,49 & 10,70 \\
\hline Conyza bonariensis (L.) Cronquist & 4,21 & 5,84 & 10,05 \\
\hline Tabernaemontana angulata Mart. ex Müll. Arg. & 4,21 & 4,55 & 8,75 \\
\hline Phyllanthus sp. & 2,68 & 5,84 & 8,52 \\
\hline Spermacoce verticillata $\mathrm{L}$. & 3,63 & 4,55 & 8,18 \\
\hline Myrcia sp. & 3,63 & 3,90 & 7,53 \\
\hline \multicolumn{4}{|c|}{ Scleria pterota var. melaleuca (Rchb. ex Schltdl. \& Cham.) } \\
\hline Uittien & 3,82 & 3,25 & 7,07 \\
\hline Spermacoce exilis (L.O. Williams) C.D. Adams & 2,87 & 3,90 & 6,76 \\
\hline Davilla rugosa Poir. & 3,44 & 3,25 & 6,69 \\
\hline Scoparia dulcis $\mathrm{L}$. & 3,82 & 2,60 & 6,42 \\
\hline Doliocarpus major J.F. Gmel. & 2,10 & 2,60 & 4,70 \\
\hline Inga alba (Sw.) Willd. & 2,10 & 2,60 & 4,70 \\
\hline Morfotipo 2 & 1,34 & 2,60 & 3,94 \\
\hline Galipea trifoliata Aubl. & 1,72 & 1,95 & 3,67 \\
\hline Stryphnodendron guianense (Aubl.) Benth. & 1,72 & 1,95 & 3,67 \\
\hline Byrsonima aerugo Sagot & 0,96 & 2,60 & 3,55 \\
\hline Miconia alata (Aubl.) DC. & 1,53 & 1,95 & 3,48 \\
\hline Myrciaria tenella (DC.) O. Berg. & 1,53 & 1,30 & 2,83 \\
\hline Vismia guianensis (Aubl.) Pers. & 1,53 & 1,30 & 2,83 \\
\hline Dichapetalum rugosum (Vahl) Prance & 1,15 & 1,30 & 2,45 \\
\hline Morfotipo 1 & 1,15 & 1,30 & 2,45 \\
\hline Morfotipo 3 & 0,76 & 1,30 & 2,06 \\
\hline Pilocarpus sp. & 0,96 & 0,65 & 1,61 \\
\hline Cyperus sp. & 0,57 & 0,65 & 1,22 \\
\hline Euphorbia heterophylla L. & 0,57 & 0,65 & 1,22 \\
\hline Gustavia augusta L. & 0,38 & 0,65 & 1,03 \\
\hline Morfotipo 4 & 0,38 & 0,65 & 1,03 \\
\hline Tetracera willdenowiana Steud. & 0,19 & 0,65 & 0,84 \\
\hline Total & 100 & 100 & 200 \\
\hline
\end{tabular}


As espécies mais importantes para o SAF 2 (sem queima) foram Myrciaria tenella e Scleria pterota. Myrciaria tenella ocupou tal posição devido sua alta densidade (Tabela 3). Na seqüência, em ordem decrescente de espécies com maior valor de IM encontram-se Olyra glaberrima, Davilla aspera, Tabernaemontana angulata, Davilla rugosa e Spermacoce verticillata, as quais têm na densidade o seu parâmetro principal. As espécies que registraram as maiores densidades e freqüências no SAF 2 foram: Myrciaria tenella, Olyra glaberrima, Scleria pterota e Davilla aspera.

Tabela 3. Percentual dos parâmetros fitossociológicos das espécies do banco de sementes do solo nos SAF 2 (paricá, caupi e feijão de porco sem uso do fogo), Pará, Brasil (DR = Densidade Relativa, FR = Frequência Relativa, IM =Índice de Valor de Importância Modificado).

Table 3. Percentage of phytosociological parameters of species seed bank of soil in the agroforestry system 2 (S. amazonicum, V.unguiculata, and Canavalia ensiformes without the use of fire), Pará, Brazil. $(D R=$ Relative Density, $F R=$ Relative Frequency, $I M=$ Importance Value Index Modified).

\begin{tabular}{|c|c|c|c|}
\hline Espécie & DR & FR & IM \\
\hline $\begin{array}{l}\text { Myrciaria tenella (DC.) O. Berg. } \\
\text { Scleria pterota var. melaleuca (Rchb. ex Schltdl. \& }\end{array}$ & 12,00 & 6,17 & 18,17 \\
\hline Cham.) Uittien & 8,19 & 8,64 & 16,83 \\
\hline Olyra glaberrima Raddi & 8,76 & 4,32 & 13,08 \\
\hline Davilla aspera (Aubl.) Benoist & 5,14 & 4,94 & 10,08 \\
\hline Tabernaemontana angulata Mart. ex Müll. Arg. & 4,57 & 4,32 & 8,89 \\
\hline Davilla rugosa Poir. & 4,38 & 3,09 & 7,47 \\
\hline Spermacoce verticillata L. & 4,00 & 3,09 & 7,09 \\
\hline Galipea trifoliata Aubl. & 3,24 & 3,70 & 6,94 \\
\hline Croton matourensis Aubl. & 2,29 & 4,32 & 6,61 \\
\hline Solanum sp. & 2,67 & 3,70 & 6,37 \\
\hline Sabicea amazonensis Wernham & 3,05 & 3,09 & 6,13 \\
\hline Scoparia dulcis L. & 2,86 & 3,09 & 5,94 \\
\hline Clidemia hirta (L.) D. Don & 1,52 & 4,32 & 5,84 \\
\hline Panicum laxum Sw. & 2,48 & 3,09 & 5,56 \\
\hline Vismia guianensis (Aubl.) Pers. & 2,48 & 3,09 & 5,56 \\
\hline Lasiacis ligulata Hitchc. \& Chase & 2,67 & 2,47 & 5,14 \\
\hline Pilocarpus sp. & 1,52 & 2,47 & 3,99 \\
\hline Inga alba (Sw.) Willd. & 1,71 & 1,85 & 3,57 \\
\hline Gustavia augusta L. & 1,52 & 1,85 & 3,38 \\
\hline Mabea paniculata Spruce ex Benth. & 1,52 & 1,85 & 3,38 \\
\hline Doliocarpus major J.F. Gmel. & 2,10 & 1,23 & 3,33 \\
\hline Passiflora sp. & 1,33 & 1,85 & 3,19 \\
\hline Miconia minutiflora (Bonpl.) DC. & 1,90 & 1,23 & 3,14 \\
\hline Connarus sp. & 1,14 & 1,85 & 2,99 \\
\hline Urena lobata L. & 1,14 & 1,85 & 2,99 \\
\hline Morfotipo 3 & 1,71 & 1,23 & 2,95 \\
\hline Scleria sp. & 1,71 & 1,23 & 2,95 \\
\hline Cecropia sp. & 1.33 & 1,23 & 2,57 \\
\hline Rhynchospora sp. & 1,33 & 1,23 & 2,57 \\
\hline Astrocaryum gynacanthum Mart. & 1,14 & 1,23 & 2,38 \\
\hline Senna latifolia (G. Mey.) H.S. Irwin \& Barneby & 1,14 & 1,23 & 2,38 \\
\hline Inga sp. & 0,76 & 1,23 & 2.00 \\
\hline Miconia sp. & 0,76 & 1,23 & 2.00 \\
\hline Urena sp. & 0,76 & 1,23 & 2,00 \\
\hline Morfotipo 4 & 0,38 & 1,23 & 1,62 \\
\hline Pavonia malacophylla (Link \& Otto) Garcke & 0,95 & 0,62 & 1,57 \\
\hline Myrcia fallax (Rich.) DC. & 0,76 & 0,62 & 1,38 \\
\hline Byrsonima spicata (Cav.) DC. & 0,57 & 0,62 & 1,19 \\
\hline Abarema jupumba (Willd.) Britton \& Killip & 0,38 & 0,62 & 1,00 \\
\hline Attalea maripa (Aubl.) Mart. & 0,38 & 0,62 & 1,00 \\
\hline Dalbergia sp. & 0,38 & 0,62 & 1,00 \\
\hline Lacistema floribundum Miq. & 0,38 & 0,62 & 1,00 \\
\hline Lygodium venustum Sw. & 0,38 & 0,62 & 1,00 \\
\hline Stryphnodendron guianense (Aubl.) Benth. & 0,38 & 0,62 & 1,00 \\
\hline Paspalum sp. & 0,19 & 0,62 & 0,81 \\
\hline Total & 100 & 100 & 200 \\
\hline
\end{tabular}


As espécies mais relevantes no SAF 3 são as pertencentes à família Cyperaceae (Serjania paucidentata e Cyperus laxus) seguida de Porophyllum ruderale e Myrciaria tenella (Tabela 4). Cyperus laxus garantiu a segunda posição em termos de IM, em função de seus elevados valores de densidade e frequência.

Tabela 4. Percentual dos parâmetros fitossociológicos das espécies do banco de sementes do solo no SAF 3 (paricá, açaí e cupuaçu com o uso do fogo), Pará, Brasil. (DR = Densidade Relativa, $\mathrm{FR}=$ Frequência Relativa, IM = Índice de Valor de Importância Modificado).

Table 4. Percentage of phytosociological parameters of species seed bank of soil in the agroforestry system 3 (S. amazonicum, E. oleracea, and T. grandiflorum with the use of fire), Pará, Brazil. $(D R=$ Relative Density, $F R=$ Relative Frequency, $I M=$ Importance Value Index Modified).

\begin{tabular}{lccc}
\hline \multicolumn{1}{c}{ Espécie } & DR & FR & IM \\
\hline Serjania paucidentata DC. & 12,33 & 8,39 & 20,72 \\
Cyperus laxus Lam. & 12,10 & 8,39 & 20,49 \\
Porophyllum ruderale (Jacq.) Cass. & 9,59 & 6,99 & 16,58 \\
Myrciaria tenella (DC.) O. Berg. & 6,62 & 8,39 & 15,01 \\
Davilla aspera (Aubl.) Benoist & 8,68 & 6,29 & 14,97 \\
Urena sp. & 7,08 & 7,69 & 14,77 \\
Sabicea amazonensis Wernham & 3,65 & 5,59 & 9,25 \\
Mimosa annularis Spruce ex Benth. & 4,34 & 4,90 & 9,23 \\
Scleria pterota var. melaleuca (Rchb. ex Schltdl. \& Cham.) & & & \\
Uittien & 4,34 & 4,90 & 9,23 \\
Uncaria guianensis (Aubl.) J.F. Gmel. & 3,65 & 4,90 & 8,55 \\
Doliocarpus brevipedicellatus Garcke & 2,97 & 3,50 & 6,46 \\
Smilax schomburgkiana Kunth & 2,97 & 3,50 & 6,46 \\
Urena lobata L. & 3,42 & 2,80 & 6,22 \\
Ravenala guyannensis (Rich.) Steud. & 1,83 & 4,20 & 6,02 \\
Bauhinia macrostachya Wall. & 2,97 & 2,80 & 5,77 \\
Passiflora nitida Kunth & 2,05 & 3,50 & 5,55 \\
Rhynchanthera hispida Naudin & 2,05 & 2,10 & 4,15 \\
Coutoubea spicata Aubl. & 1,60 & 2,10 & 3,70 \\
Talisia carinata Radlk. & 2,05 & 1,40 & 3,45 \\
Scoparia dulcis L. & 1,14 & 2,10 & 3,24 \\
Byrsonima aerugo Sagot. & 0,91 & 2,10 & 3,01 \\
Pilocarpus sp. & 1,60 & 1,40 & 3,00 \\
Solanum asperum Rich. & 0,91 & 0,70 & 1,61 \\
Morfotipo 13 & 0,68 & 0,70 & 1,38 \\
Morfotipo 11 & 0,46 & 0,70 & 1,16 \\
Total & 100 & 100 & 200 \\
\hline
\end{tabular}

No SAF 4, Myrciaria tenella destacou-se com maior IM $(19,93)$, ocupando tal posição por seus elevados valores de densidade e frequência, seguida de Davilla rugosa e Arrabidea bilabiata (Tabela 5). No que diz respeito à frequência relativa destacaram-se Myrciaria tenella, Arrabidea bilabiata, Tabernaemontana angulata e Connarus sp.

Tabela 5. Percentual dos parâmetros fitossociológicos das espécies do banco de sementes do solo no SAF 4 (paricá, açaí e cupuaçu, sem uso do fogo). Pará, Brasil. ( $D R=$ Densidade Relativa, $\mathrm{FR}=$ Frequência Relativa, IM = Índice de Valor de Importância Modificado).

Table 5. Percentage of phytosociological parameters of species seed bank of soil in the agroforestry system 4 (S. amazonicum, E. oleraceae, and T. grandiflorum without the use of fire), Pará, Brazil. ( $D R=$ Relative Density, $F R=$ Relative Frequency, $I M=$ Importance Value Index Modified).

\begin{tabular}{lccc}
\hline \multicolumn{1}{c}{ Espécie } & DR & FR & IM \\
\hline Myrciaria tenella (DC.) O. Berg. & 12,83 & 7,10 & 19,93 \\
Davilla rugosa Poir. & 8,02 & 5,46 & 13,48 \\
Continua... & & & \\
\hline
\end{tabular}




\section{Continuação Tabela 5}

Arrabidaea bilabiata (Sprague) Sandwith

6,61

6,21

Connarus sp.

Tabernaemontana angulata Mart. ex Müll. Arg.

Spermacoce verticillata $\mathrm{L}$.

Inga alba Wild.

Croton matourensis Aubl.

Galipea trifoliata Aubl.

Scleria pterota var. melaleuca (Rchb. ex Schltdl. \& Cham.)

Uittien

Clidemia hirta (L.) D. Don

Phenakospermum guyannense (Rich.) Endl.

Memora allamandiflora Bureau ex K. Schum.

Lasiacis ligulata Hitchc. \& Chase

Attalea maripa (Aubl.) Mart.

Senna latifolia (G. Mey.) H.S. Irwin \& Barneby

Myrcia fallax (Rich.) DC.

Mandevilla sp.

Mabea angustifolia Spruce ex Benth.

Davila sp.

Davilla aspera (Aubl.) Benoist

Rhynchospora rubber sp.

Dalbergia sp.

Paulinia sp.

Sabicea amazonensis Wernham

Abarema cochleata (Willd.) Barneby \& J.W. Grimes

Myrcia cuprea (O. Berg) Kiaersk.

Gustavia augusta L.

Miconia minutiflora (Bonpl.) DC.

Myrcia sp.

Platonia insignis Mart.

Solanum sp.

Total geral
4,61

5,61

5,61

5,41

4,41

4,41

2,81

2,40

2,81

2,61

2,00

2,40

2,61

2,00

1,40

1,80

1,80

1,60

1,40

1,60

1,60

1,40

1,20

1,00

0,60

0,40

0,40

0,40

100
6,56

6,01

6,56

5,46

5,46

4,92

3,83

3,83

3,28

3,28

2,73

2,73

3,28

2,73

2,19

2,19

2,73

2,19

2,19

2,19

2,19

1,64

1,64

1,64

1,64

1,09

1,09

1,09

0,55

0,55

100
13,17

12,22

11,17

11,08

11,08

10,33

8,23

8,23

6,08

5,68

5,54

5,34

5,28

5,14

4,79

4,19

4,14

3,99

3,99

3,79

3,59

3,24

3,24

3,04

2,84

2,09

1,69

1,49

0,95

0,95

200

\section{DISCUSSÃO}

A ocorrência do fogo mostrou-se ser um fator determinante na perda de densidade, riqueza e diversidade florística dos bancos de sementes. O efeito da queima sobre a densidade de sementes, provavelmente foi influenciado pelo período de permanência da área sob a ação do fogo e pela temperatura atingida. $O$ fogo também ocasionou diminuição da densidade e da riqueza do banco de sementes de Floresta Estacional Semidecidual (MELO et al. 2007). Nos estudos realizados em banco de sementes na Costa Rica foi constatada redução de $50 \%$ do banco de sementes após a queima, sendo a riqueza também reduzida (EWEL et al. 1981). LEAL et al. (2006) ao avaliarem o banco de sementes em sistemas de produção com queima, também observaram redução da diversidade de espécies nas áreas que foram queimadas, fato atribuído à destruição das sementes pelo fogo.

Os valores de densidade de sementes encontrados no presente estudo são menores àqueles encontrados em outros agroecossistemas amazônicos (ARAÚJO et al. 2001, COSTA et al. 2009, MÔNACO et al. 2003), inclusive quando comparados com sistemas agroflorestais da região (COSTA \& MITJA 2009). Essas diferenças de densidade de sementes entre os outros estudos e a presente pesquisa podem ser explicadas, em parte, pelos intervalos de avaliação da germinação das amostras na casa de vegetação. Os intervalos entre as avaliações podem não ter sido suficientemente curtos para acompanhar todas as germinações dos propágulos oriundos do banco de sementes do solo dos sistemas agroflorestais estudados. Quanto à riqueza de espécies, porém, os bancos de sementes do solo dos sistemas avaliados neste estudo assemelham-se àqueles encontrados em sistemas agroflorestais no Amazonas, cujos valores variaram de 26 a 44 (COSTA \& MITJA 2009).

As formas de vida predominantes nos bancos de sementes das áreas de sistemas agroflorestais preparadas com fogo foram as ervas e lianas. Após a ação do fogo nos agroecossistemas estudados por 
LEAL et al. (2006), verificou-se, também, o predomínio de espécies herbáceas nos bancos de sementes. Estes resultados seguem a tendência apontada por estudos realizados por MELO \& DURIGAN (2010). Estes autores, analisando o impacto do fogo em comunidades vegetais observaram elevado número de espécies de lianas em áreas que sofreram queima. As espécies de ervas e lianas estão aptas a suportar condições climáticas adversas, tolerar altas temperaturas e ambientes secos, apresentando grande capacidade de produzir sementes (CHRISTOFFOLETI \& CAETANO 1998). Provavelmente, não é somente a ocorrência da queima que pode afetar a composição das espécies, mas também a intensidade do fogo. COCHRANE \& SCHULZE (1999) observaram que o aumento da intensidade do fogo elevou a abundância de lianas. Em solos da caatinga submetidos a queimadas controladas, as espécies herbáceas também predominaram no banco de sementes (42,8\%) (MAMEDE 2003).

MELO et al. (2007) também verificaram baixa densidade de sementes de espécies arbóreas nos bancos de sementes em áreas queimadas. XAVIER (2010), ao analisar a influência do fogo em áreas de cerrado com diferentes históricos de incêndios, constatou que as espécies herbáceas apresentam uma recuperação muito mais rápida no banco de sementes do que as lenhosas após distúrbios. GARWOOD (1989) ao estudar diversos bancos de sementes de áreas perturbadas em regiões tropicais encontrou alta quantidade de espécies herbáceas. MÔNACO et al. (2003) observaram que cerca de $36 \%$ das espécies encontradas no banco de sementes de uma floresta secundária de oito anos de sucessão no estado do Amazonas eram herbáceas. Esta mesma tendência foi observada na Amazônia Central por COSTA \& MITJA (2009) em sistemas agroflorestais e por COSTA et al. (2009) em cultivos de mandioca. A recuperação pós-distúrbio apresentada pela maioria das espécies herbáceas (principalmente gramíneas) se dá, principalmente, pelo alto potencial competitivo dessas espécies. As altas taxas de crescimento apresentadas por essas espécies, aliadas a redução da biomassa do solo ocasionada pelo fogo as levam a competir de maneira muito efetiva com as espécies arbóreas (MELO \& DURIGAN 2010, QUINTANAASCENCIO \& MORALES-HERNÁNDEZ 1997). Por isso, são tão comuns em áreas alteradas e vegetação secundária (GARWOOD 1989).

A redução das espécies de hábito arbóreo nos bancos de sementes das áreas que foram manejadas com fogo deve-se, provavelmente, ao fato de que as herbáceas (principalmente gramíneas) dificultam a instalação e o desenvolvimento das espécies arbóreas, impedindo (ou pelo menos dificultando) a produção e dispersão de seus propágulos. Deve ser levado em consideração, também, que as sementes de espécies arbóreas provavelmente são menos resistentes a ação do fogo quando comparadas às herbáceas e lianas. Isso pode ser corroborado pelos estudos realizados por BRINKMANN \& VIEIRA (1971). Esses autores, ao avaliarem o efeito do fogo na germinação de diferentes espécies, constataram uma redução na germinação de sementes arbóreas quando expostas ao fogo. Estas diferenças quanto à forma de vida das espécies são consequência do histórico de uso da terra bem como de alterações sofridas pela vegetação, assim, os resultados confirmam a hipótese de que o fogo influencia na proporção da forma de vida no banco de sementes, criando condições favoráveis ao surgimento de espécies herbáceas.

As famílias que se destacaram na presente pesquisa também foram observadas em outros estudos na Amazônia, por exemplo, COSTA \& MITJA (2009) ao estudar o banco de sementes de solos em sistemas agroflorestais do Amazonas observaram que Euphorbiaceae e Asteraceae foram as famílias de maior riqueza (seis espécies cada). Em cultivos de mandioca da Amazônia Central, as famílias com maior riqueza foram Asteraceae com sete espécies, seguida de Euphorbiaceae com quatro (COSTA et al. 2009). Em termos de tamanho de populações (número de indivíduos), as famílias mais representativas do banco de sementes dos sistemas agroflorestais de Bragança foram Borraginaceae, Poaceae, Cyperaceae, Asteraceae e Euphorbiaceae.

Analisados em conjunto, o número de famílias nos SAF's que sofreram ação do fogo foi menor do que nas áreas de SAF's que não foram manejadas com fogo. Dentre os SAF's localizados em áreas que foram preparadas com fogo, a família Cyperaceae foi a mais importante. O aumento na germinação das espécies dessa família provocado pelo fogo também foi observado e constatado por CARDOSO et al. (2000). Características como a quebra de dormência das sementes facilitada pelo calor e a capacidade de rebrotamento podem favorecer a colonização inicial por determinados grupos de plantas. Aliado a isso, a falta de cobertura do solo proporciona maior potencial de entrada de sementes na área através da chuva de sementes (THOMPSON 1992). Resultados semelhantes foram encontrados por GUARIGUATA \& OSTERTAG (2001) e LEAL et al. (2006) que verificaram a predominância de espécies herbáceas nos estágios iniciais de áreas que sofreram ação do fogo.

Algumas espécies foram exclusivas das áreas preparadas com fogo, por exemplo, Conyza bonariensis e Spermacoce exilis, corroborando com os resultados encontrados por IKEDA et al. (2008). Por 
outro lado, a ausência de Lasiacis ligulata e Panicum laxum, nos bancos de sementes dos SAF's queimados, pode indicar que o fogo tenha inviabilizado as sementes dessas espécies (KISSMANN \& GROTH 1997). Da mesma forma, Croton matourensis não ocorreu no banco de sementes das áreas que sofreram queima. MELO et al. (2007) não constataram a presença de Croton floribundus no banco de sementes das áreas que sofreram queima. Segundo estes autores, as sementes dessa espécie arbórea pioneira podem ter sido eliminadas pelo fogo.

\section{CONCLUSÃO}

O fogo causa modificações na estrutura e na composição florística do banco de sementes em sistemas agroflorestais, reduzindo a riqueza, diversidade e densidade do banco de sementes desses agroecossistemas.

O fogo influencia na proporção da forma de vida no banco de sementes, criando condições favoráveis ao surgimento de espécies herbáceas e lianas. Desta forma, os sistemas agroflorestais apresentam maior suscetibilidade ao desenvolvimento e propagação de vegetação espontânea, quando expostos ao fogo, sobretudo de espécies herbáceas e lianas.

\section{AGRADECIMENTOS}

Ao Conselho Nacional de Desenvolvimento Científico e Tecnológico (CNPq) por conceder a bolsa de doutorado para a primeira autora.

\section{REFERÊNCIAS}

ARAÚJO MM et al. 2001. Densidade e composição florística do banco de sementes do solo de florestas sucessionais na região do Baixo Rio Guamá, Amazônia Oriental. Scientia Forestalis 59: 115-130.

AULD TD \& DENHAM AJ. 2006. How much seed remains in the soil after a fire? Plant Ecology 187: 15-24.

BRINKMANN WLF \& VIEIRA AN. 1971. The effect of burning on germination of seeds at different soil depths of various tree species. Turrialba 21: 77-82.

CARDOSO EL et al. 2000. Composição e dinâmica da biomassa aérea após a queima em savana gramíneo-lenhosa no Pantanal. Pesquisa Agropecuária Brasileira 35: 2309-2316.

COCHRANE MA \& SCHULZE MD. 1999. Fire as a recurrent event in tropical forests of the eastern Amazon: effects on forest structure, biomass and species composition. Biotropica 31: 2-16.

COSTA JR \& MITJA D. 2009. Bancos de sementes de plantas daninhas em sistemas agroflorestais na Amazônia Central. Revista Brasileira de Ciências Agrárias 4: 298-303.

COSTA JR et al. 2009. Bancos de sementes de plantas daninhas em cultivos de mandioca na Amazônia Central. Planta Daninha 27: 665-671.

CHRISTOFFOLETI PJ \& CAETANO RSX. 1998. Soil seed banks. Scientia Agricola 55: 74-78.

EWEL J et al. 1981. Slash and burn impacts on a Costa Rican wet forest site. Ecology 62: 816-829.

GARWOOD NC. 1989. Tropical soil seed banks: a review. In: LECK MA et al. Ecology of soil seed banks. San Diego: Academic Press. p.149-209.

GERHARDT K \& HYTTEBORN H. 1992. Natural dynamics and regeneration methods in tropical dry forests - an introduction. Journal of Vegetation Science 3: 361-364.

GUARIGUATA MR \& OSTERTAG R. 2001. Neotropical secondary forest succession: changes in structural and functional characteristics. Forest Ecology and Management 148: 185-206.

IBGE. 1983. Instituto Brasileiro de Geografia e Estatística. Bragança, Norte, Pará. Coleção de Monografias Municipais. Nova Série 17: 1-16.

IKEDA FS et al 2008. Banco de sementes em cerrado sensu stricto sob queimada e sistemas de cultivo. Pesquisa Agropecuária Brasileira 43: 667-673.

KEITH DA. 1996. Fire-driven extinction of plant populations: a synthesis of theory and review of evidence from Australian vegetation. Proceedings of the Linnean Society of New South Wales 116: 37-78.

KISSMANN KG \& GROTH D. 1997. Plantas infestantes e nocivas. Tomo I. 2.ed. São Paulo: BASF. 824p.

LEAL EC et al. 2006. Banco de sementes em sistemas de produção de agricultura com queima e sem queima no município de Marapanim, Pará. Boletim do Museu Paraense Emílio Goeldi. Ciências Naturais 1: 19-29.

LORENZI H. 2006. Manual de identificação e controle de plantas daninhas: plantio direto e convencional. 6.ed. Nova Odessa: Instituto Plantarum. 339p.

LOUZADA JNC et al. 2003. O fogo como instrumento de manejo em agroecossistemas. Informe Agropecuário 24: 2936.

MAGURRAN AE. 1988. Ecological diversity and its measurement. London: Croom Helm. 179 p.

MAMEDE MA 2003. Efeito do manejo agrícola tradicional sobre o banco de sementes do solo em uma área de caatinga, município de Sobral, CE. Dissertação (Mestrado em Desenvolvimento e Meio Ambiente). Fortaleza: UFC. 68p.

MELO ACG et al. 2007. Efeito do fogo sobre o banco de sementes em faixa de borda de Floresta Estacional Semidecidual, SP, Brasil. Acta Botanica Brasilica 21: 927-934. 
MELO ACG \& DURIGAN G. 2010. Impacto do fogo e da regeneração da comunidade vegetal em borda de Floresta Estacional Semidecidual (Gália, SP, Brasil). Revista Brasileira de Botânica 33: 37-50.

MÔNACO LM et al. 2003. Banco de sementes de uma floresta secundária amazônica dominada por Vismia. Acta Amazonica 33: 41-52.

MUELLER-DOMBOIS D \& ELLENBERG H. 1974. Aims and methods of vegetation ecology. New York: John Wiley and Sons. $547 \mathrm{p}$.

NOBREGA AMF et al. 2009. Banco de sementes de remanescentes naturais e de áreas reflorestadas em uma várzea do Rio Mogi-Guaçu - SP. Revista Árvore 33: 403-411.

PARKER VT et al. 1989. Pattern and process in the dynamics of seed banks. In: LECK M et al. (Eds.). Ecology of Soil Seed Banks. San Diego: Academic Press. p.367-384.

QUINTANA-ASCENCIO PF \& MORALES-HERNÁNDEZ M. 1997. Fire-mediated effects of shrubs, lichens, and herbs on the demography of Hypericum cumulicola in patchy Florida scrub. Oecologia 112: 263-271.

SCHIMTZ MC 1992. Banco de sementes no solo em áreas do reservatório da UHE Paraibuna. In: KAGEYAMA PY. Recomposição da vegetação com espécies arbóreas nativas em reservatórios de usinas hidrelétricas da CESP. Piracicaba: IPEF. p.7-8.

THOMPSON K. 1992. The functional ecology of seed banks. In: FENNER M. ed. Seeds: The ecology of regeneration in plant communities. Wallingford: CAB International. p.231- 258.

THOMPSON K \& GRIME JP. 1979. Seasonal variation in the seed banks of herbaceous species in ten contrasting habitats. Journal Ecology 67: 893-921.

UHL C \& BUSCHBACHER R. 1985. A disturbing synergism between cattle ranch burning practices and selective tree harvesting in the eastern Amazon. Biotropica 17: 265-268.

UHL C et al. 1981. Early plant sucession after cutting and burning in the upper Rio Negro region of the amazon basin. Journal of Ecology 69: 631-649.

WHELAN RJ et al. 2002. Critical life cycles of plants and animals: developing a process-based understanding of population changes in fireprone landscapes. In: BRADSTOCK RA et al. (Eds.) Flammable Australia: the fire regimes and biodiversity of a continent. Cambridge: Cambridge University Press. p.94-124.

XAVIER RO. 2010. Influência do fogo no banco de sementes em áreas de cerrado com diferentes históricos de incêndios. Dissertação (Mestrado em Ecologia e Recursos Naturais). São Carlos: UFSCar. 105p. 